\title{
Introduction of Cardiac Magnetic Resonance Imaging in Kosovo: First Fifty Consecutive Patients Registry Report
}

\author{
Ted Trajcheski $^{1 *}$, Lulzim Brovina ${ }^{1}$, Biljana Zafirova² ${ }^{2}$, Lada Trajceska ${ }^{3}$ D \\ ${ }^{1}$ American Hospital Pristina, Pristina, Kosovo; ${ }^{2}$ Department of Anatomy, Medical Faculty, University Ss. Cyril and Methodius, \\ Skopje, Macedonia; ${ }^{3}$ Department of Nephrology, University Clinic of Nephrology, Medical faculty, University Ss. Cyril and \\ Methodius, Skopje, Macedonia
}



\section{Introduction}

Magnetic resonance imaging (MRI), especially cardiac magnetic resonance (CMR), is fundamental to healthcare [1], particularly for cardiovascular diagnosis and treatment. It is the fastest growing imaging modality at $12 \%$ annually [2]. Overall, MRI is an extremely safe imaging modality, with over 40 million scans in USA and 300 million scans performed worldwide to date.

This imaging modality has specialized sequences [3] which have particular application for the cardiovascular system in the field of the ischemic heart disease (IHD), heart failure, and cardiomyopathy, valvular heart disease, cardiac masses and pericardial disease, diseases of the aorta, adult congenital heart diseases, and magnetic resonance angiography. In respect of the IHD, CMR is the current gold standard for the recognition of infarcted myocardium and the assessment of global and regional cardiac wall motion abnormalities.

The utility of CMR arises in the context of myocardial infarction (MI) and ischemia. It provides valuable information which may not be available from other diagnostic tools such as echocardiography and nuclear cardiology that currently dominate non-invasive diagnosis in patients with coronary artery disease (CAD). Assessment of ventricular function and mass offers reproducible results which are well validated for measuring the left ventricular (LV) and right ventricular (RV) volumes and mass. This makes it valuable for the assessment of fundamental parameters of cardiac function as well as longitudinal follow-up of patients over time. Myocardial perfusion [4], [5] CMR follows the effect of a first pass of a bolus of intravenous gadolinium through multiple planes of the myocardium using ultrafast sequences such as Fast Low Angle Shot, steady-state free precession, which can allow entire images to be acquired in $<200 \mathrm{~ms}$. Ml can be detected with high accuracy and sensitivity using late gadoliniumenhanced CMR [6]. Gadolinium (0.1-0.2 mmol/kg) is given intravenously and after $6-10 \mathrm{~min}, \mathrm{CMR}$ is commenced using an inversion recovery sequence, where the inversion time is chosen to null myocardial signal. Because normal myocardium is uniformly tightly packed with muscle, and gadolinium is an extracellular contrast agent, there is uniformly low signal in the normal 
heart. In areas of MI, the extracellular compartment is expanded, and in addition, gadolinium wash out from these areas is slow. This leads to a higher gadolinium concentration on the late enhancement scan, which shows as bright signal, and has led to the aphorism "bright is dead." Because CMR has a high resolution, it is possible to determine the transmural distribution resolution of $\mathrm{Ml}$ in vivo. The technique has been extensively validated in animal $\mathrm{Ml}$ models and has now replaced other CMR techniques for detecting $\mathrm{MI}$. In humans, late gadolinium enhanced CMR has been shown to accurately detect both $Q$-wave and non- $Q$ wave MI [7]. Because the technique is so sensitive, CMR has been shown to identify sub-endocardial MI when wall motion and perfusion by Single-photon emission computed tomography (SPECT) are normal.

The cardiomyopathies include a variety of diseases where the primary pathology directly involves the myocardium excluding CAD. CMR has proved increasingly valuable in the identification and management of these conditions. Hypertrophic cardiomyopathy requires an accurate diagnosis, determination of the distribution of hypertrophy and its functional consequences, and assessment of the likelihood of sudden death and progression to heart failure. LV hypertrophy is an important independent risk factor for cardiac events. Left ventricular noncompaction has become more recognized [8] and appears to have autosomal dominant inheritance. The morphological and functional abnormalities of dilated cardiomyopathy are clearly demonstrated and quantified by CMR.

Valvular heart disease is a field where the low cost, flexibility, and ease of handling make transthoracic echocardiography (TEE) the primary clinical tool for evaluation of valvular heart disease [9]. Moreover, TEE is superior to CMR in assessment of valve morphology and detection of small and rapidly moving vegetation's attached to the valves in endocarditis. However, CMR may play a complementary role when transthoracic acoustic windows are poor and a TEE approach is undesirable, or when results of echocardiography and catheterization are conflicting.

CMR is very safe and no long-term ill effects have been demonstrated. Anxiety and claustrophobia may be problematic in about $2 \%$ of patients [8], but mild anxiolysis is often effective. One of the most important safety issues for CMR is the prevention of introduction into the scanner area of ferromagnetic objects which can become projectiles. Metallic implants such as hip prostheses, prosthetic heart valves, coronary stents, and sternal sutures present no hazard since the materials used are not ferromagnetic (although an artifact local to the implant may be present). Care is required in patients with many cerebrovascular clips and specialist advice is needed for such patients [10]. Patients with pacemakers, implanted cardioverter defibrillators, retained permanent pacemaker leads, and other electronic implants are not scanned in every institution. It is of paramount importance to check every individual implant whether it is MRI conditional. There is a progress towards manufacturing and scanning CMRcompatible devices.

In Kosovo as worldwide, computed tomography (CT) and ultrasound have been the workhorse imaging modalities in cardiology. While the advantages of these modalities have afforded them a prominent position in cardiac imaging, MRI is promising because it directly addresses the shortcomings of both modalities CMR provides excellent soft tissue contrast without giving any ionizing radiation dose to the patient. It possesses the ability to do functional assessment of the heart for various conditions with metrics such as LV and RV systolic function and volume, as well as wall motion. Its main use is in assessment of anatomy, function and viability of the heart, but it is also useful in detecting ischemia and infarction, and in assessing congenital heart disease, the etiology of heart failure, heart valve dysfunction, masses, and the presence of inherited diseases. Limitations of CMR are considered to be different. One is the access to cardiac MRI. This can vary depending on geographical health service and patient referral policies. A limited number of specialized cardiac MRI imaging facilities, especially in rural regions, makes MRI more difficult to access than more traditional modalities, such as echocardiography and exercise stress tests. Cardiac MRI is not currently capable of high-resolution imaging of the coronary arteries and quantification of arterial stenosis, for which catheter angiography and CT angiography are superior. It does have an accepted role in assessment of anomalous coronary arteries.

\section{Objective}

The objective of the study was to study the cardiovascular magnetic resonance in the "American Hospital - Prishtina" in Kosovo with providing a more specific cardiovascular diagnosis and aid in accurate determination, or exclusion, of cardiac pathology. This enhancement to standard of care would optimize decision-making, adequate treatment, swift improvement of the health condition, and reduce mortality.

\section{Methods}

Our institution obtains a Siemens MRI scanner with static magnetic field of 1.5 Tesla. The hydrogen nucleus (a single proton) behaves as a small spinning magnet which aligns itself parallel to an external magnetic field and processes about the field in the same way that a spinning top processes in a gravitational field. The 
frequency of precession is $63 \mathrm{MHz}$ for a field strength of 1.5 Tesla which is in the radiofrequency range. Resistive gradient coils within the bore of the magnet produce the gradient fields, and the currents within these coils are driven by the gradient amplifiers. The performance of the gradient system determines how fast magnetic resonance acquisition can be. A radiofrequency coil (antenna) is coupled to a radiofrequency amplifier to excite the patient with the radiofrequency pulses, and this (or another more localized surface coil) is coupled to the receiver to measure the signals coming from the patient. A computer is required to control the scanner and generate the images. Images are then displayed in static, dynamic (cine) modes, or as multi-planar reconstructions.

The CMR imaging scan was performed by a radiology technician. The supervision, all the readings quality image assessment were done by certified MRI specialist - cardiologist.

The quality criteria were derived from multicenter studies [11] and were validated with multicenter registry data [12]. Criteria are modified from Klinke et al. [12]. The maximum score is 9, which would indicate severe artifacts in three slices or more. In respect of artifacts severe, intermediate and minor were classified, as presented in Table 1. In one patient, 20-23 MRI sequences were performed and the percentage of the three classes of artifacts was calculated from the overall number of sequences per patient.

Table 1: Quality criteria in respect of artifacts



The overall quality image assessment per patient (Table 2) was scaled ranging from poor to excellent (quantified 0-5). The assessment was performed at the single surgeon's discretion.

Table 2: Quality image assessment per patient scale

\begin{tabular}{ll}
\hline Image quality & Grade \\
\hline Excellent & 5 \\
Very good & 4 \\
Good & 3 \\
Acceptable & 2 \\
Poor & 1 \\
Very poor & 0 \\
\hline
\end{tabular}

\section{Patients}

CMR was indicated by cardiologists. Check for any contraindication for MR examination, stress study, and contrast administration, was mandatory. An informed consent for the MR examination was obtained. Limitations for performance of the CMR scan consisted of: Patient's size match with the scanner tunnel; patient anxiety; presence of metallic implants with different degree of conditionality; capacity of the patient to maintain horizontal position in the scanner; and performance of breath holds and chronic kidney disease Stages IV and V. Contraindications for the CMR scan included: Patient did not consent for the scan; metallic implants non-compatible with the CMR; and limited contraindications in patients allergic to gadolinium contrast agent or other pharmacological agents required during the procedure.

Patients were advised on fasting before examination. Intake of caffeine-containing food and beverages, theophylline or dipyridamole at least $24 \mathrm{~h}$ before adenosine study; when dobutamine study was to be performed, beta-blocker and nitrates were to be avoided. Patients were educated and provided practice on the breathing instructions. Headphones were provided. Patients skin of the chest and/or abdomen was prepared for optimal attachment of electrodes for cardiac and respiratory gating. The best comfortable supine position of the patients was obtained. The arms were placed above the head to decrease wrap artifact. It was acceptable to put the arms along the side or crossed over the chest if the patient could not tolerate arms overhead for extended periods of time. In case of Stress study 2 cubital venous lines were inserted - one for contrast and the other for adenosine or dobutamine. A minor dose of sedative was administered before procedure to improve patient's comfort during the exam. Adenosine stress: Pre-procedure check for contraindication for adenosine stress test was done. This included known hypersensitivity to adenosine, suspected bronchoconstrictive or bronchospastic disease, heart block, systemic arterial hypotension, and recent myocardial infarction. An informed consent was obtained. Electrocardiogram (ECG) and venous line prepared. During the procedure oxygen was provided, control of blood pressure and monitoring of vital signs. Medications for antagonizing the effect of adenosine and the clinical events were at immediate disposal. Postprocedure post-stress ECG was performed and final vital signs before discharge were recorded. Patients were given detailed explanation before the realized scan procedure. Medical history and examination were performed by the referring doctor and notes taken by the radiology technician as well. A written informed consent was obtained before the scan. The degree of patient anxiety was noted. Antianxiety medications such as oral or intravenous diazepam were to be administrated to achieve required relief from patient's discomfort and still maintain adequate alertness. Patients with conditional metallic implants were not excluded from the CMR scan. For that purpose, detailed patient history was taken with detailed information of the implant in consideration. Magnetic compatibility was assessed by consulting reference online documentation for each specific implant. We had patients with intracardiac implants such as different types of stents and metallic prosthetic valves. Extracardiac implants most commonly were hip and knees prothesis and metallic dental implants. 
Mainly no interference was noted by these implants except some magnetic reverberation from the metallic prosthetic valves.

Some patients required adjusting different scan settings to improve the quality of the scan and reduce interference. Most of our patients, before the scan were trained to hold their breaths in order to reduce scanning motion artifacts. Few patients, although having kept their breath well, during the procedure experienced random misses that required repeating the sequence. We had a tendency to perform all procedures with breath holds, but sometimes it was impossible and free breathing was allowed. This resulted in overall satisfactory quality of the scan in all our cases.

\section{Results}

CMR in Kosovo was first performed in September 2019. Since then fifty CMR scans have been performed and interpreted in the "American Hospital Prishtina." The age of the patients ranged from 17 to 82 and the number of male and female patients was well balanced (Table 3).

Table 3: Patients demographics, assessed cardiac conditions, advanced tests

\begin{tabular}{ll}
\hline Parameters & $\mathrm{n}(\%)$ \\
\hline $\mathrm{n}=50$ & $25(50)$ \\
Male & $50.62 \pm 13.56$ \\
Age (years) & \\
MRI assessment for & $19(38)$ \\
IHD & $17(34)$ \\
Structural heart disease [14] & $12(24)$ \\
Cardiomyopathies [15] & $2(4)$ \\
Great vessels pathology [16] & $0(0)$ \\
Congenital heart disease & $12(24)$ \\
Adenosine stress MRI perfusion test & $4(33)$ \\
Positive stress adenosine MRI perfusion test & $46(92)$ \\
LGE & $15(30)$ \\
Positive LGE & \\
\hline MRI: Magnetic resonance imaging, LGE: Late gadolinium enhancement, IHD: Ischemic heart disease.
\end{tabular}

It was possible to perform the CMR scan in all patients. Different cardiac pathologies were encountered in the examined patients. The most common was IHD - 19 (38\%). It presented as stable ischemia with preserved heart structure and function, patients with past myocardial infarction and various degree of LV impairment as well as ischemic myocardial injury (Picture 1). Patients inquired for structural heart disease were $17(34 \%)$ and cardiomyopathies were 12 $(24 \%)$. In two (4\%) patients, the CMR was indicated because of a disease of the aorta of pulmonary artery. Out of all performed CMR, only in $5(10 \%)$ the diagnosis was not confirmed. Late gadolinium enhancement was performed in $46(92 \%)$ of CMR procedures and a positive finding of cardiac fibrosis was shown in 15 $(30 \%)$ patients.

The adenosine test was performed in 12 $(24 \%)$ of patients and 4 of them (33\%) were positive on ischemia (Picture 2).
Among all cases, two patients were diagnosed for history of myocarditis, two for hypertrophic obstructive cardiomyopathy (HOCM), one with thrombus in the left atrium, one with shunt of the pulmonary artery; ASD, two with pleural, and one with pericardial effusion (Picture 3).

Considering the MRI conditional implants (Table 4), six (12\%) patients had stents, 2 (4\%) by-pass, and other two $(4 \%)$ had mechanical aortic valve. One patient refused only the performance of gadolinium test, and $2(4 \%)$ had significant anxiety with the need of extra sedation. In $6(12 \%)$ patients, the estimated glomerular filtration rate (eGFR) below $30 \mathrm{ml} / \mathrm{min}$ prevented us from using gadolinium contrast agent. No other relative contraindications were met. During and after CMR imaging procedure no adverse events occurred.

Table 4: Assessed patients with relative contraindications: presence of MRI conditional implants, anxiety and declined eGFR

\begin{tabular}{ll}
\hline Patients with relative MRI contraindications & $\mathrm{n}=18(37 \%)$ \\
\hline MRI conditional implants & \\
Coronary stent(s) & $6(12)$ \\
Aorto-coronary by-pass & $2(4)$ \\
Mechanical/bio valve & $2(4)$ \\
Anxiety with a need of additional sedation & $2(4)$ \\
eGFR $<30 \mathrm{ml} / \mathrm{min} / 1.73 \mathrm{~m}^{2}$ & $6(12)$ \\
\hline MRI: Magnetic resonance imaging, eGFR: Estimated glomerular filtration rate. &
\end{tabular}

In respect of artifacts classification (Table 5), in all 962 performed MRI sequences, we experienced severe artifacts as ghosting (Picture 4) in only one patient, intermediate artifacts (Picture 5) in four patients, and minor artifacts were present in five patients. Most of the minor artifacts were due to respiratory drift and metallic implants (Picture 6).

Table 5: Quality criteria in respect of artifacts in all performed sequences

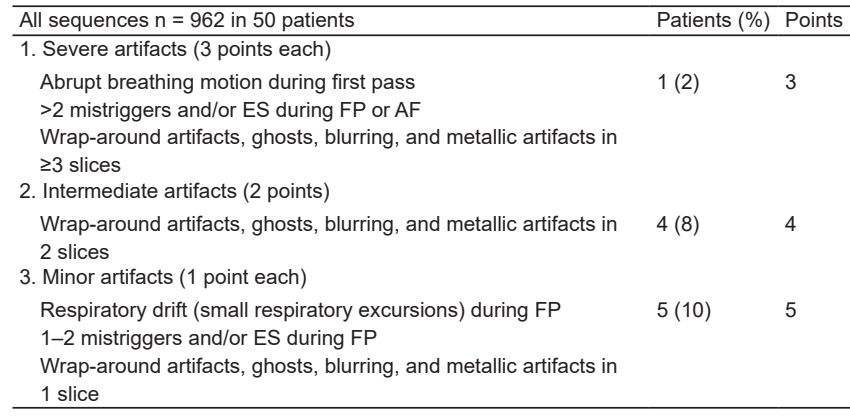

The overall quality image assessment per patient (Table 6) was scaled ranging from poor to excellent (quantified 0-5). There were no poor and very poor assessments. Least was acceptable $(2 \%)$ and excellent (4\%). Most of the assessments were very good and good graded $(46 \%$ and $48 \%$, respectively).

Table 6: Quality image assessment per patient

\begin{tabular}{lll}
\hline Image quality per patient & Grade & $\mathrm{n}=50(\%)$ \\
\hline Excellent & 5 & $2(4)$ \\
Very good & 4 & $23(46)$ \\
Good & 3 & $24(48)$ \\
Acceptable & 2 & $1(2)$ \\
Poor & 1 & 0 \\
Very poor & 0 & 0 \\
\hline
\end{tabular}






Picture 1: (a) Late gadolinium enhancement delineating myocardial fibrosis due to past myocardial infarction in the apical inferolateral region short axis view, (b) mid and apical anterior region - 2 chamber view, (c) subendocardial fibrosis in mid inferior segment, respectively
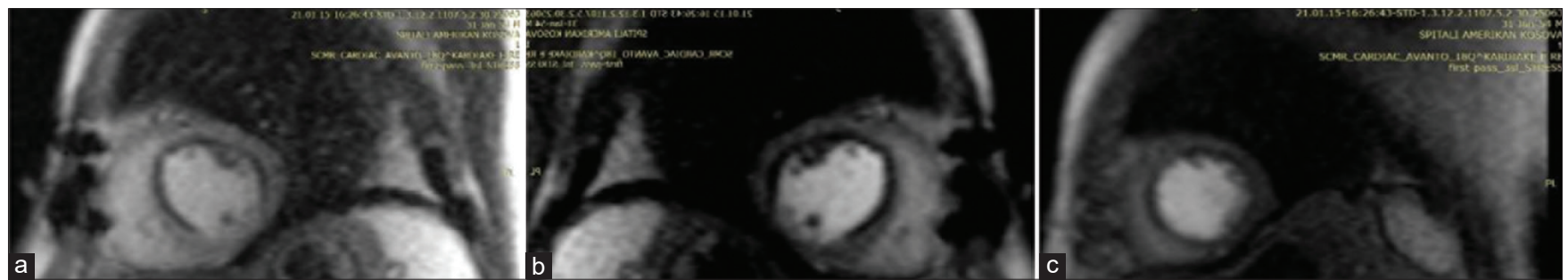

Picture 2: Adenosine stress myocardial perfusion imaging shows impaired vascularization seen by darker colorization of the subendocardium Gadolinium contrast agent has reduced penetration in the regions with impaired blood flow in different segments (a-c) Vasodilating effect is absent in the stenotic blood vessels (a) basal segment, (b) mid segment, (c) apical segment
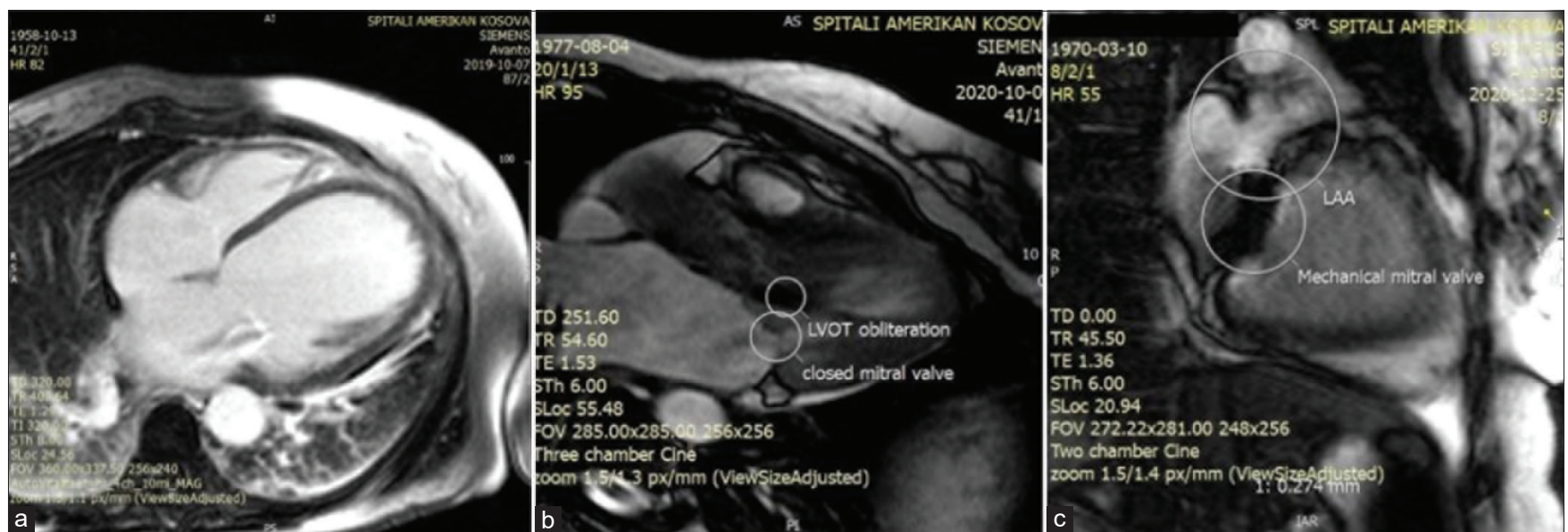

Picture 3: (a) Dilated CMP post-myocarditis presenting enlarged LV with reduced contractility, thinned LV wall. (b) HOCM with obliterated LVOT. Mitral valve leaflets are closed in end systole with simultaneous obliteration of the LVOT. Severe concentric thickening of the basal and mid LV wall. (c) Metallic mitral valve, LAA. LAA shows absence of thrombus allowing safe DC cardio version. CMP: Cardiomyopathy; LV: Left ventricle; HOCM: Hypertrophic obstructive cardiomyopathy; LVOT: Left ventricular outflow tract; LAA: Left atrial appendage, DC: Direct current

\section{Discussion}

By establishing a CMR center in Kosovo, we are bringing close to the patients this valuable imaging procedure. It will help in the assessment of: The ischemic disease of the heart, myocardial perfusion and viability; quantification of ventricular structure and function; adult valvular and structural abnormalities of the heart; congenital disease of the heart or great vessels; assessment of tumor masses of the heart; abnormality of the thoracic aorta; and evaluation of infiltrative diseases of the heart muscle, such as sarcoidosis, amyloidosis, hemochromatosis, and Fabry disease; assessment of diseases of the pericardium; exclusion of anomalous coronary origins; and quantification of cardiovascular shunts [13]. 


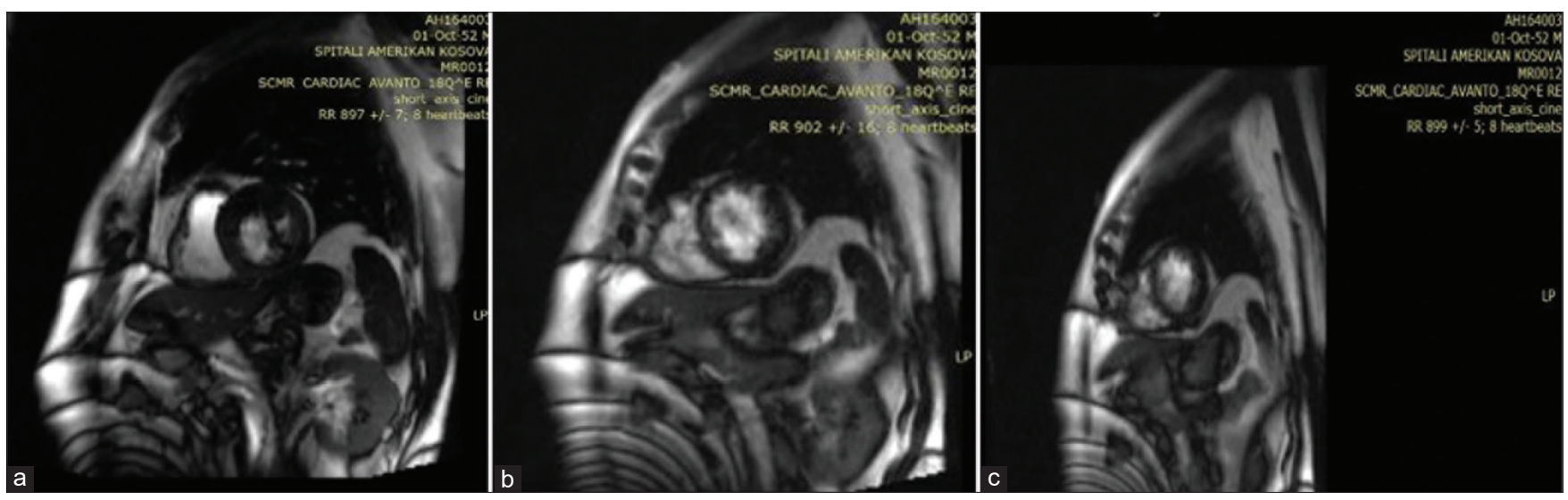

Picture 4: Severe artifacts presented as ghosting artifact in three slices in one patient cardiac magnetic resonance imaging



Picture 5: (a) Intermediate artifacts presented by mechanical mitral valve; (b) motion artifact at aortic and mitral mechanical valves; (c) respiratory ghosting; (d) frequency artifact in four chamber view going from LV to RV; (e) frequency artifact in short axis view going from $L V$ to $R V$



Picture 6: Minor artifacts presented as: Respiratory motion artifacts in Late gadolinium enhancement 4 chamber view (a) and short axis view (b), wrap-around effect of patient's back (c) 
In our report, we have presented the implementation of CMR procedure with all the complexity of its performance. We obtained patients' information, education, and informed consent. Safety issues were addressed with protocols considering procedural risks, absolute, and relative contraindications [10]. Among the first 48 consecutively assessed patients, we covered several cardiac pathologies. Most of them were ischemic and structural heart diseases, which is in line with other publications [17], [20]. It was expected since it represents the most common cardiac pathology. CMR has shown to be valuable asset in establishing valvular disease, shunts, and even thrombotic masses in atria which were the platform for further treatment of our patients. Those conditions are already described as pathologies where CMR is more precise than conventional diagnostic tools [9], [21], [22]. Echocardiography [23] appears to be more readily available in assessment of the RV and LV function and structure and likely to be better tolerated. Echo is better able to assess valvular function compared to MRI. It can be limited by poor acoustic windows and difficultly in viewing some regions of the heart, sometimes requiring trans-esophageal echo.

Considerations for alternative imaging tests in specific indications are echocardiography, myocardial perfusion scan, and coronary angiography. Regarding cardiac viability and MRI, the alternative from the side of the echocardiogram is dependent on the expertise of the cardiologist performing and interpreting the echocardiogram. Stress sestamibi or thallium myocardial nuclear perfusion scan is wellestablished clinically to provide physiological rather than anatomical information [4], [5], [11] but does lack spatial resolution. Nuclear medicine has lower spatial resolution than $\mathrm{MRI}$, but is widely used to assess heart muscle function. In our study, perfusion test was performed in $24 \%$ of patients and provided valuable information on myocardial viability, positive in $33 \%$ of those. We acknowledged patients with myocardial fibrosis due to history of myocarditis and also HOCM [24]. Exercise stress test has lower accuracy and not suited to all patient populations, for example, poor exercise tolerance due to arthritis or pulmonary disease. MRI angiogram is as well obtained with CT angiography [25]. The main disadvantages are: The radiation dose required for the study, and inability to quantify flow (e.g., valvular regurgitant fraction and shunt calculation) as well as iodinated contrast, which is usually mandatory and might be deleterious in patients with a poor renal function. Soft-tissue contrast is less prominent in CT than in MRI scans. CT offers excellent spatial resolution and better demonstration of calcification. Both CT and MRI might be contraindicated in the setting of renal impairment or allergy to iodinated contrast or gadolinium, respectively. In our study, safety protocol was obtained, providing information on patients with low eGFR in consultation with nephrologist, and those patients were not endured by this procedure, as advised by many authors [10], [14]. We performed it in most of our patients and found it positive in $30 \%$ of them, which was as high as in many other studies [26].

Echocardiography as a safe and more readily available procedure offers only limited views of the aorta and great vessels. Catheter angiography by all means is invasive, with a greater risk of complications. It offers the best spatial resolution and potential for endovascular intervention if appropriate. It can be difficult to perform/interpret in the setting of complex anatomy, in the absence of prior cross-sectional imaging [19]. We also performed successful assessment of great blood vessels and gained valuable information for those patients.

Considering the relative contraindications, we followed the protocol, informed the patients and did not detain from MRI assessment. Patients with MRI conditional [15], [16], [27] pacemakers, stents, metallic valvulae, and by pass were not refused for the procedure, as in other institutions with big flow of patients. Individual implants were reference checked in the large MRI safety database. Furthermore, patients were well assessed before the procedure in respect of tolerability and anxiety. Additional sedation and excellent results were obtained from the procedure in $5 \%$ of patients. In other reports, the crucial communication factor between the doctor, the patient, and strategy for obtaining full procedure result has been stressed [8], [28].

At present, MRI is extensively used for the evaluation of cardiovascular and thoracic disorders because of the well-established advantages that include use of non-ionizing radiation, good contrast, and high spatial resolution. Despite the advantages of this technique, numerous categories of artifacts are frequently encountered [29], [30].

In line with the data of these previous studies; in our results, we have shown the same most frequently present artifacts as respiratory drifts (small respiratory excursions), wrap-around artifacts, ghosts, blurring, and metallic artifacts. We performed 20-23 MRI sequences per patient, resulting in overall 960 sequences. Non pathologic variations were discussed with anatomist. We closely monitored the appearance of artifacts during the sessions. To obtain better quality results, we repeated sequences with lower quality until getting satisfactory image quality. Furthermore, the quality image assessment per patient was at quite high level, which obtained the necessary diagnostic input required for more precise diagnosing and better treatment.

\section{Conclusion}

Over the last year, the landscape of cardiac imaging in Kosovo has changed dramatically. MRI has 
emerged as an excellent imaging tool for diagnosing and characterizing a nearly exhaustive spectrum of heart diseases. This new method provides clinicians with substantial additional information, including tissue characterization, accurate measurements of ventricular volumes, and function. Importantly, MRI (as the slightly more mature cardiac imaging modality) has recently made dramatic strides in the evaluation of IHD. For the moment, it seems reasonable to consider CMR when ventricular function, tissue characterization, or blood flow physiology are the principal clinical concerns. If detailed anatomical evaluation is the major clinical focus, invasive coronary angiogram remains the gold standard. This procedure is not reimbursed by the public health coverage fund. To provide more patients with invaluable diagnosis, this imaging procedure should have public funding. It would bring net reduction in public expenses by providing accurate diagnosis and timely treatment to cardiovascular patients.

\section{AQ5 References}

1. Shah S, Chryssos ED, Parker H. Magnetic resonance imaging: A wealth of cardiovascular information. Ochsner J. 2009;9(4):266-77.

PMid:21603453

2. Bhuva AN, Moralee $\mathrm{R}$, Moon $\mathrm{JH}$, Manistry $\mathrm{CH}$. Making $\mathrm{MR}$ available for patients with cardiac implantable electronic devices: Growing need and barriers to change. Eur Radiol. 2020;30:1378-84. https://doi.org/10.1007/s00330-019-06449-5 PMid:31776746

3. Kramer CM, Barkhausen J, Bucciarelli-Ducci C, Flamm SD, Ki RJ, Nagel E. Standardized cardiovascular magnetic resonance imaging (CMR) protocols: 2020 update. J Cardiovasc Magn Reson. 2020;22(1):17. https://doi.org/10.1186/ s12968-020-00607-1

\section{PMid:32089132}

4. Shehata ML, Basha TA, Hayeri MR, Hartung D, Teytelboym OM, Vogel-Claussen J. MR myocardial perfusion imaging: Insights on techniques, analysis, interpretation, and findings. Radiographics. 2014;34(6):1636-57. https://doi.org/10.1148/ rg.346140074

PMid:25310421

5. Hamirani YS, Kramer CM. Cardiac MRI assessment of myocardial perfusion. Future Cardiol. 2014;10(3):349-58. https://doi.org/10.2217/fca.14.18

PMid:24976472

6. Doltra A, Amundsen BH, Gebker R, Fleck E, Kelle S. Emerging concepts for myocardial late gadolinium enhancement MRI. Curr Cardiol Rev. 2013;9(3):185-90. https://doi.org/10.2174/15 $73403 \times 113099990030$ PMid:23909638

7. Greenwood JP, Motwani M, Maredia N, Brown JM, Everett CC Nixon $\mathrm{J}$, et al. Comparison of cardiovascular magnetic resonance and single-photon emission computed tomography in women with suspected coronary artery disease from the clinical evaluation of magnetic resonance imaging in coronary heart disease (CE-MARC) Trial. Circulation. 2014;129(10):1129-38. https://doi.org/10.1161/circulationaha.112.000071

\section{PMid:24357404}

8. Munn Z, Pearson A, Jordan Z, Murphy F, Pilkington D, Anderson A. Patient anxiety and satisfaction in a magnetic resonance imaging department: Initial results from an action research study. J Med Imaging Radiat Sci. 2015;46(1):23-9. https://doi.org/10.1016/j.jmir.2014.07.006

PMid:31052060

9. Mathew RC, Löffler Al, Salerno M. Role of cardiac magnetic resonance imaging in valvular heart disease: Diagnosis, assessment, and management. Curr Cardiol Rep. 2018;20(11):119. https://doi.org/10.1007/s11886-018-1057-9 PMid:30259253

10. Ghadimi M, Sapra A. Magnetic resonance imaging contraindications. In: Stat Pearls. Treasure Island, FL: Stat Pearls Publishing; 2020

11. Wolff SD, Schwitter J, Coulden R, Friedrich MG, Bluemke DA Biederman RW, et al. Myocardial first-pass perfusion magnetic resonance imaging: A multicenter dose-ranging study. Circulation. 2004;110(6):732-7. https://doi.org/10.1161/01. cir.0000138106.84335.62 PMid:15289374

12. Klinke V, Muzzarelli S, Lauriers N, Locca D, Vincenti G, Monney P, et al. Quality assessment of cardiovascular magnetic resonance in the setting of the European CMR registry: Description and validation of standardized criteria. J Cardiovasc Magn Reson. 2013;15(1):55. https://doi.org/10.1186/1532-429x-15-55 PMid:23787094

13. Dickstein K. Clinical Utilities of Cardiac MRI an Article from the E-Journal of the ESC Council for Cardiology Practice. Vol. 6; 2008. Available from: https://www.escardio.org/journals/ejournal-of-cardiology-practice/volume-6/clinical-utilities-ofcardiac-mri. [Last accessed on 2021 Apr 10]

14. Lohrke J, Frenzel T, Endrikat J, Alves FC, Grist TM, Law M, et al 25 years of contrast-enhanced MRI: Developments, current challenges and future perspectives. Adv Ther. 2016;33(1):1-28. https://doi.org/10.1007/s12325-015-0275-4 PMid:26809251

15. Ainslie M, Miller C, Brown B, Schmitt M. Cardiac MRI of patients with implanted electrical cardiac devices. Heart. 2014;100(5):3639. https://doi.org/10.1136/heartjnl-2013-304324 PMid:23872585

16. Irnich W, Irnich B, Bartsch C, Stermann W, Gufler H, Weiler G. Do we need pacemakers resistant to magnetic resonance imaging? Europace. 2005;7(4):353-65. https://doi.org/10.1016/j. eupc.2005.02.120

PMid: 15944094

17. Steinberg $\mathrm{DH}$, Staubach S, Franke D, Sievert H. Defining structural heart disease in the adult patient: Current scope, inherent challenges and future directions. Eur Heart J Suppl. 2010;12(9):2-9. https://doi.org/10.1093/eurheartj/suq012

18. Lüscher TF. Cardiomyopathies: Definition, diagnosis, causes, and genetics. Eur Heart J. 2016;37(23):1779-82. https://doi. org/10.1093/eurheartj/ehw254

PMid:27317096

19. Bamalan OA, Soos MP. Anatomy, thorax, heart great vessels In: Stat Pearls. Treasure Island, FL: Stat Pearls Publishing; 2020.

20. Laspas F, Pipikos T, Karatzis E, Georgakopoulos N, Prassopoulos V, Andreou J, et al. Cardiac magnetic resonance versus single-photon emission computed tomography for detecting coronary artery disease and myocardial ischemia: Comparison with coronary angiography. Diagnostics (Basel). 2020;10(4):190. https://doi.org/10.3390/diagnostics10040190 PMid:32235380

21. Lopez-Mattei JC, Shah DJ. The role of cardiac magnetic 
resonance in valvular heart disease. Methodist Debakey Cardiovasc J. 2013;9(3):142-8. https://doi.org/10.14797/ mdcj-9-3-142

PMid:24066197

22. Seewöster T, Hilbert S, Spampinato RA, Hahn J, Hindricks G, Bollmann A, et al. Tumor or thrombus? The role of cardiac magnetic resonance imaging in differentiating left atrial mass in a transplanted heart: A case report. J Atr Fibrillation. 2017;10(4):1608. https://doi.org/10.4022/jafib.1608 PMid:29487675

23. Thavendiranathan $P$, Dickerson JA, Scandling $D$, Balasubramanian V, Pennell ML, Hinton A, et al. Comparison of treadmill exercise stress cardiac MRI to stress echocardiography in healthy volunteers for adequacy of left ventricular endocardial wall visualization: A pilot study. J Magn Reson Imaging. 2014;39(5):1146-52. https://doi.org/10.1002/jmri.24263 PMid:24123562

24. Hansen MW, Merchant N. MRI of hypertrophic cardiomyopathy: Part I, MRI appearances. AJR Am J Roentgenol. 2007;189(6):1335-43. https://doi.org/10.2214/ajr.07.2286 PMid:18029869

25. Sakuma H. Coronary CT versus MR angiography: The role of MR angiography. Radiology. 2011;258(2):340-9. https://doi. org/10.1148/radiol.10100116

PMid:21273518

26. Weinreb JC, Rodby RA, Yee J, Wang C, Fine D, McDonald R, et al. Use of intravenous gadolinium-based contrast media in patients with kidney disease: Consensus statements from the American college of radiology and the national kidney foundation. Radiology. 2021;298(1):28-35. https://doi. org/10.1148/radiol.2020202903

PMid:33170103

27. Shellock FG, Woods TO, Crues JV $3^{\text {rd }}$. MR labeling information for implants and devices: Explanation of terminology. Radiology. 2009;253(1):26-30. https://doi.org/10.1148/radiol.2531091030 PMid:19789253

28. Dziuda $\measuredangle$, Zieliński P, Baran P, Krej M, Kopka L. A study of the relationship between the level of anxiety declared by MRI patients in the STAI questionnaire and their respiratory rate acquired by a fibre-optic sensor system. Sci Rep. 2019;9(1):4341. https:// doi.org/10.1038/s41598-019-40737-w

PMid:30867494

29. Alfudhili K, Masci PG, Delacoste J, Ledoux BJ, Bercheier G, Dunet $\mathrm{V}$, et al. Current artefacts in cardiac and chest magnetic resonance imaging: Tips and tricks. $\mathrm{Br} J$ Radiol. 2016;89(1062):20150987. https://doi.org/10.1259/ bjr.20150987

PMid:26986460

30. Ferreira PF, Gatehouse PD, Mohiaddin RH, Firmin D. Cardiovascularmagneticresonanceartefacts. JCardiovasc Magn Reson. 2015;15(1):41. https://doi.org/10.1186/1532-429x-15-41 PMid:23697969

Author Queries???

AQ5: Kindly cite Reference 18 in the text part and also cite in chronological order 